\title{
Construct this! $O$ por qué el constructivismo sistémico es real
}

Dr. Aldo Mascareño

Doctor en Sociología, Universidad de Bielefeld

Profesor investigador Escuela de Gobierno, Universidad Adolfo Ibáñez

aldo.mascareno@uai.cl

Resumen

El presente artículo desarrolla la tesis de que el constructivismo sistémico se sostiene en operaciones reales de comunicación entre individuos reales. El resultado de esto es la emergencia de constelaciones simbólicas y sistemas sociales reales cuyas consecuencias son también reales para los mismos individuos.

\section{Abstract}

The present article argues that the systemic constructivism is supported by real operations of communication between real individuals. The result of this is the emergency of symbolic constellations and real social systems, which consequences are also real for the same individuals.

Palabras Clave: Constructivismo, teoría de sistemas, operaciones, realidad, observación

Keywords: Constructivism, systems theory, operations, reality, observation

\section{Introducción}

Operar significa existir Niklas Luhmann

El problema del constructivismo ha sido generalmente abordado como la antítesis del realismo, del objetivismo y la ontología. Derivar de ello formulaciones siempre fáciles del tipo 'la realidad no existe, es una invención', 'todo depende del observador', 'nadie está en lo correcto, todos tienen razón', es el resultado más inmediato de una comprensión demasiado rápida del problema. El énfasis de la teoría de sistemas en la autonomía de las acciones y vivencias individuales y en la autonomía de las constelaciones estructurales y simbólicas de los sistemas sociales, así como en la continuidad operativa y la emergencia de la comunicación, impiden soluciones unilaterales que condensen la problemática en fórmulas más propias de los medios de comunicación que de la reflexividad científica. Del mismo modo, la concentración de la explicación del constructivismo sistémico en la modalidad de observación de segundo orden como observación de observaciones, no debe hacer olvidar que lo que ella observa son observaciones de primer orden, y que no hay nada más real para el observador de primer orden que aquello que observa en su entorno, es decir, en el horizonte de su mundo.

La hipótesis de este artículo es que el constructivismo sistémico se sostiene en las operaciones reales de comunicación entre individuos reales para quienes la operación 
misma es inobservable. El resultado de esto es la emergencia de constelaciones simbólicas y sistemas sociales reales cuyas consecuencias son también reales para los mismos individuos. Solo luego de ello se puede suponer constructivismo. Prueba de esto es que antes del big bang de la operación psíquica y social, los dinosaurios no tenían nombre; para esto fue necesario operar, observar, distinguir e indicar. O dicho algo más técnicamente: los individuos vivencian sus acciones y las acciones y vivencias de otros de modos diferenciados por la aplicación de esquemas diferenciados de observación sustentados en operaciones reales; los sistemas sociales y constelaciones simbólicas modulan esas diferencias en estructuras y semánticas temporalmente estabilizadas y generan a la vez esquemas de observación alternativos disponibles para la operación psíquica y social. Es decir, acciones y vivencias, por un lado, estructuras y constelaciones simbólicas, por otro, se sostienen sobre la realidad de las operaciones psíquicas y comunicacionales. Condición de posibilidad de cualquier rendimiento constructivista son, por tanto, esas operaciones reales; o dicho de otro modo: construct this!, pues ellas son el punto ciego de la comunicación, la garantía de la existencia del mundo y no dependen del observador.

Para desplegar este argumento, quiero en primer lugar indagar en el estatuto de realidad de las operaciones sistémicas (1) para luego especificar esto a nivel del sistema psíquico (2) y del sistema comunicacional (3), y con esto mostrar cómo ellas contribuyen a la emergencia de estructuras y constelaciones simbólicas cuyo estatuto de realidad constituye lo social (4). Concluyo con un breve paralelo entre las modalidades racionalista, realista y constructivista de teorización social (5).

\section{Construct this!: la operación sistémica}

Quiero partir por una afirmación común en la sociología: sin individuos no hay sociedad. Ella puede ser encontrada en diversas formulaciones en Durkheim (1985), Simmel (1992), Weber (1992), Parsons (1970), Luhmann (2007). No parece ser una sentencia controversial, por tanto es un buen punto de partida. Ella es una afirmación, sin embargo, trivial, por un lado, y una llena de presupuestos, por otro. El primer presupuesto es que hay individuos y hay sociedad, es decir, que son niveles ('de sistema', se podría decir en teoría de sistemas) distintos. El segundo indica que hay una relación entre individuo y sociedad. Y el tercero señala la topicidad de esa relación: los individuos son el sustento de la sociedad. Interesante es también ilustrar lo que la afirmación no indica, esto es, que la sociedad no puede ser reducida a los individuos (primer presupuesto: hay individuos y sociedad), tampoco que ellos son sus componentes (segundo presupuesto: existe una relación y para ello debe haber dos términos autónomos e irreductibles), ni que los individuos producen la sociedad (tercer presupuesto: son su sustento, pero la relación de causalidad no se especifica positivamente).

Si se trata entonces de niveles autónomos y relacionados, se debe explicar qué es lo que en cada caso constituye su autonomía (esto es: su estatuto de realidad) y cuál es la dirección de la causalidad entre ellos. Como afirmación general, se puede indicar lo siguiente: la autonomía del sistema es siempre producida por un tipo especial (distinto) de operación que le otorga unidad al sistema y que lo hace observable como tal y observador a la vez. Es decir, el sistema opera, y en la operación se constituye la observación. Operación significa en este contexto la transformación de los elementos del sistema en otros elementos de la misma cualidad continuamente; observación en tanto, implica distinguir e indicar qué elemento específico es seleccionado para la continuidad de la operación. Autopoiesis indica la unidad de esta diferencia (Luhmann 2005a). 
Operación y observación son simultáneas, pero la operación es fundamental: es, por decirlo así, la base material del sistema. A este resultado se puede llegar del siguiente modo: la existencia del mundo como horizonte de posibilidades significativas de selección solo es posible a condición de una operación de distinción que indique al mundo. Pero para que esta opción emerja, la materialidad del mundo tiene que necesariamente anteceder; tiene que existir un ultramundo anterior al mundo como posibilidad de selección significativa. Sin materialidad, esto es, sin mundo físicoquímico y sin mundo orgánico en el sentido de Parsons (1978, 2007), la actividad no emerge: no hay individuos ni sociedad. El sentido de los sistemas psíquicos y sociales, es decir, el mundo significativo, solo puede existir como mundo sobre un ultramundo que afirme y sostenga su continuidad, su actividad, y que dé a los sistemas de sentido la garantía de que cada mundo se sostiene sobre un ultramundo que es el mismo para todos (mundo empírico), a pesar de que sea inaccesible para la observación y a pesar de que esta indique posibilidades distintas en distintos casos (ver Figura 1).

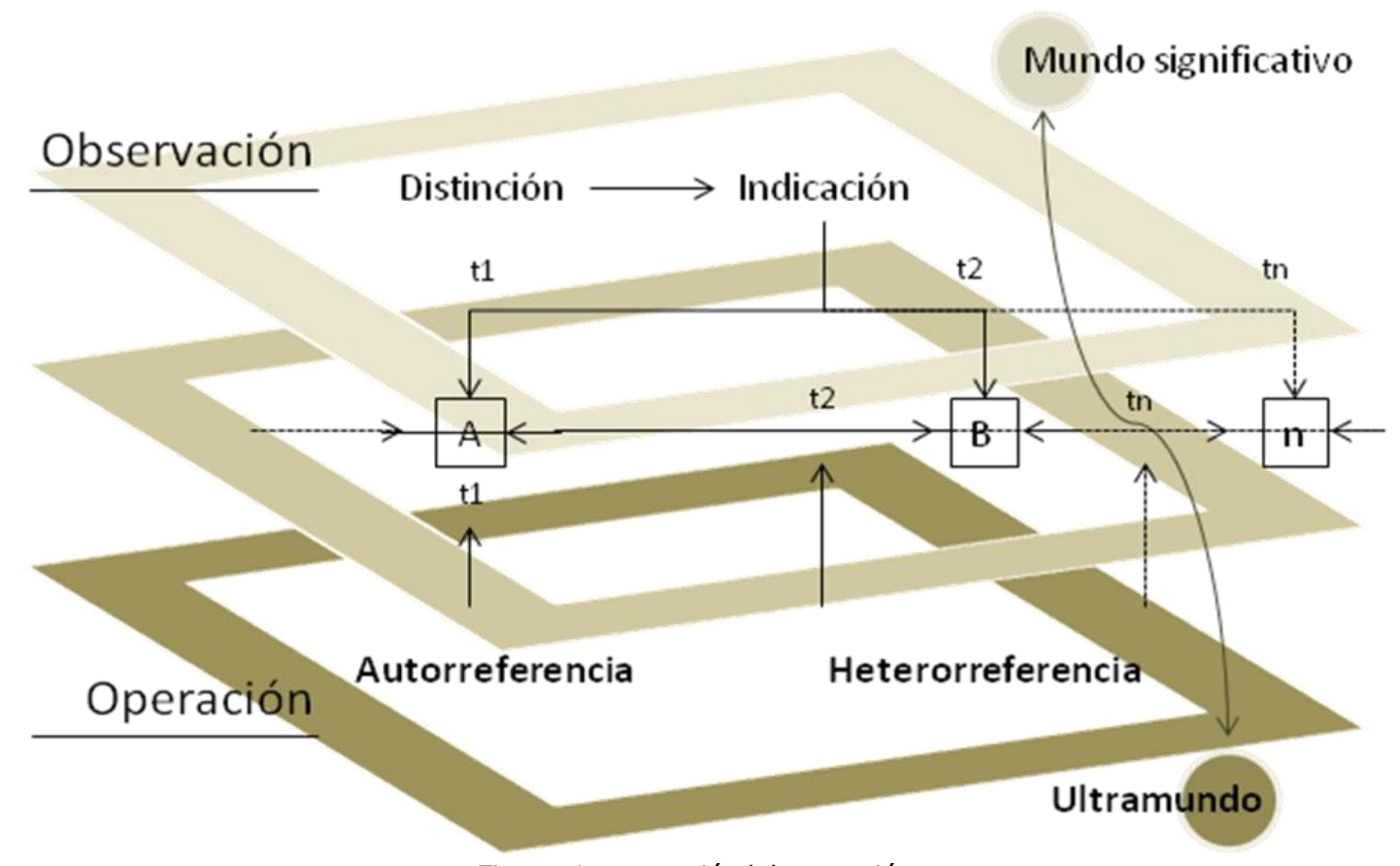

Figura 1: operación/observación

Dicho de otro modo, el medium significativo mundo puede ser mundo significativo solo si se efectúa la operación de distinción. Lo que emerge es producto de la operación, primero como distinción entre operación y observación, y luego por la re-entry de esta distinción en sí misma como diferencia entre autorreferencia y heterorreferencia. Esta última distinción está en la base de la clausura operativa del sistema - de la conciencia o de la comunicación- y no indica más que el hecho de que las observaciones o remiten a sí mismas o remiten a otras -al mismo u otro cogitans en el caso de los sistemas psíquicos, a la misma u otra comunicación en el caso de los sistemas sociales.

Si esta dinámica se detiene, cada cogitación de la conciencia o cada comunicación de la sociedad se vería en poco tiempo sobrecargada de sí misma y colapsaría la autopoiesis del sistema. Por ello, tanto la conciencia como la comunicación operan en 
eventos discretos pero continuos, uno tras otro, los que luego de su aparición desaparecen en otro evento que se observa a sí mismo o al entorno, es decir, en modalidad de autorreferencia o de heterorreferencia. En cada operación está contenida esta posibilidad de bifurcación, por tanto de bi-estabilidad de la operación y del crossing de una a otra (Luhmann 2005a). El paso siguiente no es anticipable, por esto el mundo está tan abierto a su permanencia como a su cambio, a su morfoestásis como a su morfogénesis, y solo la condensación de posibilidades de conectividad de la comunicación en sistemas sociales hacen que la reestabilización del sistema sea más visible que la variación.

De todo ello deriva que la operación (del sistema) es la garantía de la existencia de un ultramundo que puede otorgar seguridad de que la observación acontece, de que es un evento en el mundo, de que es real y no una ilusión o la intervención de un genio maligno (o benigno). Por ello la parábola de Brecht sobre una vieja controversia filosófica china puede siempre tener lugar: "mientras discutía la pregunta: '¿es real el río Amarillo, o existe únicamente en las cabezas?', el congreso de los sabios fue sorprendido por una crecida y pereció" (von Beyme 1994: 200). La operación es facticidad del ultramundo; la observación es validez del mundo para el observador. Dicho de otro modo, la operación es la ultrarealidad de la observación; la observación es real gracias a este sustento de ultrarealidad, y es a la vez libre de construir su validez (sus indicaciones) acerca del mundo, pues la operación ultrareal no indica cuál es la indicación que la observación debe seleccionar; solo le otorga a la observación la compulsión de selección. Puesto en los términos de Luhmann (2005a: 39): "No hay duda, por tanto, de que el mundo externo existe, de que es posible un contacto real con él como condición de realidad de la operación del sistema. Solo la diferencialidad de aquello que existe es sobreimaginada por el observador, y lo es en tanto con la ayuda de la especificación de distinciones, se abre un espacio de combinaciones inmensamente rico que sirve al sistema para la decisión sobre las propias operaciones". La operación (siempre del sistema) conecta la ultrarealidad del mundo (ultramundo) con el mundo de la observación, porque ella es una operación real, a la vez del ultramundo (que acopla mundo físico-químico, orgánico, sistema nervioso, sistema neuronal), y a la vez del mundo significativo de los sistemas psíquicos y sociales (esto es, el mundo de la observación, la distinción y la indicación, el mundo que co-emerge en la operación).

Bajo este esquema, la ultrarealidad del mundo es inaprensible en su inmediatez para el mundo significativo; es decir: la operación es el punto ciego de la observación, en tanto esta se dedica a distinguir e indicar. Kant (1992[1781]: 137) ya había formulado esto en los siguientes términos: "no podemos tener conocimiento de los objetos como cosas en sí, sino en tanto que son objeto de la intuición sensible, es decir, como fenómenos [...] Es preciso también que se note que aunque aquí se dice que no podemos conocer esos objetos como cosas en sí, que queda reservado que, por lo menos, pueden pensarse". De esto Kant derivó un sujeto trascendental y una ética de fundamento racionalista. Husserl (2005[1931]: 103-104) radicalizó el problema bajo la fórmula de la subjetividad trascendental: "Es claro que la verdad, o la verdadera realidad de los objetos, solo puede extraerse de la evidencia, y que ella sola es quien hace que tenga sentido para nosotros un objeto (sea de la forma o especie que quiera) 'realmente' existente, verdadero, justamente válido [...] Toda razón de ser brota de aquí; brota, pues, de nuestra subjetividad trascendental misma; toda imaginable adecuación surge como verificación nuestra, es nuestra síntesis, tiene en nosotros su último fundamento trascendental". La inobservabilidad del ultramundo está en la génesis del racionalismo kantiano y de la fenomenología husserliana, pero en ninguno de los casos la operación real de la razón trascendental o de la subjetividad 
trascendental queda simplemente supuesta - como sucedía bajo la fórmula idealista de Berkeley esse est percipi (Berkeley 2008), recurrida por Maturana y Varela para 'fundamentar filosóficamente' la autopoiesis de la observación (ver Maturana 1982).

La trascendentalidad del sujeto kantiano, del ego husserliano e incluso el realismo trascendental de Descartes, intentan sostener una garantía de conocimiento confiable que pueda modular las impresiones del ego empírico (en el sentido de Husserl). 'Trascendental' en estos casos es una metáfora que implica la designación de un campo de operaciones universalmente válido para todos, el cual no se puede postular sino por la negación de accesibilidad del ultramundo: inaccesibilidad al Ding an Sich (Kant), realidad entre paréntesis (Husserl), duda metódica (Descartes). O puesto en los términos de Luhmann (2005a: 47): "La realidad es aquello que no se conoce cuando se la conoce". La inobservabilidad del 'mundo de las cosas' es la condición del descubrimiento filosófico de la operación que hace posible observar el mundo de las cosas como fenómeno (Kant, Husserl) o como realismo trascendental (Descartes). En cualquiera de estos casos vale, sin embargo, lo siguiente: el ultramundo de la operación es lo que permite ver el mundo de las cosas. 'Trascendental' es lo que da garantía de verdad en los cuerpos filosóficos referidos; 'operación' es la garantía de realidad en el constructivismo sistémico. Ambos (lo trascendente y la operación) son condición de posibilidad de la observación del mundo y por tanto escapan a la observación; o dicho más correctamente: cuando la observación los indica, solo puede reconocer que está observando, pero precisamente esa inescapabilidad de la observación de sí misma es su garantía de continuidad del ultramundo y del mundo de la significación, pues presupone operación. La operación no se construye; la operación es, "y operar significa existir" (Luhmann 1997: 39).

Vistas las cosas de este modo, en un sentido amplio, para los objetivistas el mundo de las cosas tenía variadas posiciones y solo bastaría cambiar de posición en él para observar otra cosa. Para los subjetivistas, en tanto, había una variedad de perspectivas y solo bastaba interrogar al sujeto para conocer su perspectiva del mundo. El constructivismo sistémico, en tanto, se contenta con nada más (y nada menos) que una operación de distinción: los sistemas (psíquicos y sociales) distinguen y sus distinciones crean horizontes significativos. Las posiciones en el mundo (objetivismo) son distinguidas sobre la base de operaciones de observación que coinciden en sus distinciones y se acoplan coordinadamente (en la referencia a lugares, momentos o relaciones sociales de distinto tipo). Las perspectivas de sujetos (subjetivismo) son a su vez observadas con la ayuda de distinciones como individuo/colectivo, persona/sociedad, actor/estructura, sujeto/mundo, las que permiten paralelamente tomar en consideración la particularidad de cada perspectiva y sugerir que se trata de una pluralidad de ellas (en un colectivo, en la sociedad, en una estructura social, en el mundo). Con esta universalidad de la operación, no se afirma ningún carácter trascendental (o empírico sensible) de la operación en un sentido filosófico; más bien se indica que el constructivismo sistémico reemplaza la distinción trascendental/empírico por la distinción operación/observación, y que esta distinción goza de universalidad y realidad para sistemas psíquicos y sociales: su realidad es la realidad de la observación, y quien considere que la observación no es real, solo puede hacerlo a condición de operar con una distinción que confirma la ultrarealidad de la operación.

La operación no es una facultad de la conciencia y tampoco de la sociedad, de modo tal que se pudiera afirmar alguna naturaleza del mundo o la existencia de un suprasistema físico o metafísico desde el cual surgiera el mundo en un acto de creación o emanación. Ni la conciencia ni la sociedad existen antes de la operación, y la realidad 
de la operación no predetermina un tipo de conciencia universal o un tipo de sociedad como finalidad. Ellas son (solo) observaciones que aplican un esquema de distinción determinada; pero como tales son (también) reales. Es decir, a la operación no es inmanente una idea de perfectibilidad, por ello no puede fundarse en esto una filosofía de la historia al modo de un proyecto de completa 'transformación en sistema' de cada rincón del mundo, ni tampoco derivar de esto alguna idea escatológica de emancipación humana (aunque probablemente sí una ética de aspiración tenue como modus vivendi -ver Mascareño 2006). La operación acontece evento a evento, momento a momento: "Cada evento refiere a eventos anteriores, a expectativas y estructuras que, por cierto, son visibles solo en los eventos mismos" (Nassehi 2006: 250). No puede haber predeterminación ni predestinación en ese acontecer. Se puede sin duda intentar orientarlo, manejarlo, incluso controlarlo, por la razón o la fuerza (con los fracasos y éxitos que en ambos casos siempre se ha tenido), pero no hay en él puntos de anclaje para esperar un despliegue progresivo de un tipo específico de mundo. Quien a pesar de ello lo proponga, debe contar con que al operar es inmanente el acontecer, que no se construye por el observador, aunque sí es posible determinar las consecuencias que esa operación tiene para la construcción (real) de la observación psíquica y social.

\section{Construct this!: la operación psíquica}

La operación psíquica es un caso especial de la operación de sistemas, al igual que la comunicación lo es. Sus eventos y últimos elementos son cogitaciones y el acoplamiento de uno a otro constituye el flujo operativo de la bifurcación entre auto y heterorreferencia. A esta transformación o flujo de un elemento a otro puede llamársele autopoiesis de la conciencia. 'Flujo' o 'movimiento' son, sin embargo, metáforas que presuponen también posiciones entre las que se mueve lo que se mueve. La tradición, desde Aristóteles hasta la escolástica, supuso la independencia del cronos y el topos, como substancias respecto de las cuales se podía medir la rectitud de las lecturas del observador en tiempo y espacio. Con ello fue posible orientarlo desde afuera: desde el kosmos en los griegos, desde Dios en la escolástica. Kant (1992[1781]), en tanto, introdujo tiempo y espacio como formas de la sensibilidad propias del entendimiento. Esto permitió la autoorientación libre del sujeto en el mundo, y a la vez la promesa de una reconciliación al final de la historia del progreso humano por efecto de la perfectibilidad inmanente a la naturaleza (Kant 2002a). En la reconstrucción de la teoría de sistemas, topos y cronos aparecen como dimensiones del sentido socialmente construido por el observador: como 'esto/lo otro' (dimensión factual) y como 'antes/después' (dimensión temporal) (Luhmann 1984; ver también Stichweh 2003). Si esto es así, la posibilidad de distinguir tiempo y espacio debe también estar contenida en la operación. Dicho de otro modo: en la referencia al sí mismo (autorreferencia) y en la referencia a lo otro (heterorreferencia), la operación distingue posiciones, y el paso de una posición a otra solo puede realizarse distinguiendo entre antes y después, es decir, por medio de una construcción temporal que resulta de la propia operación. Tiempo y espacio constituyen la unidad de la diferencia de la operación de auto y heterorreferencia; un tipo de oscilación constante entre estos valores operativos. Se trata, por tanto, de formas universales, aunque no trascendentales en el sentido de que por ello pueda derivarse su objetividad para el entendimiento, aunque sí su carácter de fenómeno.

Para condensar esta relación operativa universal de posiciones y tiempo en los sistemas psíquicos, Husserl (2005: 75-76) optó por la idea de intencionalidad: "Las vivencias de la conciencia llámanse también intencionales, pero a condición de que el término de intencionalidad no signifique otra cosa que esta propiedad fundamental y 
universal de la conciencia, consistente en ser esta conciencia de algo, en llevar la conciencia en sí, en cuanto cogito, su cogitatum". 'Fundamental y universal' significa en los términos aquí empleados ultrarrealidad de la operación. Para Luhmann (2005b: 31) esta intencionalidad constituye la unidad operacional del sistema psíquico: "la conciencia es siempre una conciencia de fenómenos que se asocia a un continuo procesamiento de autorreferencia (conciencia) y heterorreferencia (fenómenos), y la intencionalidad es precisamente la forma que hace operable esa diferencia". Con esto la conciencia -el sistema psíquico- puede dejar de ser descrita como agregación de sus múltiples rendimientos: percepción, pensamiento, sentimiento, voluntad; es decir, deja de ser comprendida por los resultados de su operación intencional, y pasar a ser entendida en términos de su actividad fundamental. Esta actividad no se puede construir por el observador (construct this!); se puede distinguir mediante una diferencia entre operación y observación, pero esa propia distinción refleja la operación que subyace a la observación (como en la Figura 1), es decir, constata la imposibilidad de una creatio de la ultrarrealidad por medio de la paradoja de la observación que observa que no se puede seguir observando. Se puede decir incluso: "La unidad de la observación y su objeto (Gegenstand) no se encuentra en la condición trascendental de su posibilidad, sino - si se puede expresar de ese modo- en la condición trascendental de su imposibilidad: en el encuentro de una paradoja. Y puesto que esto cuenta igualmente para la autoobservación, se sigue de ello una base para la proyección de un alter ego" (Luhmann 2005c: 59).

Esto puede ser descrito (sin recurso a la observación) como procesualidad operativa de la conciencia, como su ultrarealidad, que hace posible observar, distinguir e indicar en el acontecer (posición) y devenir (tiempo) de la operación. Si la observación es el empleo operativo de una distinción, entonces la operación es su medium y, por ello, lo mismo puede contar para uno u otro observador, aunque lo que observen sea distinto, pues de ello se sigue la aplicación de un esquema de observación desigual así como la oscilación entre sus valores, y no la posición diferenciada de cada uno en el mundo (objetivismo) o su singularidad como sujeto (subjetivismo). Con esto, en definitiva, se asegura la autopoiesis de la conciencia: "La operación intencional es una constante oscilación entre heterorreferencia y autorreferencia, y evita de ese modo que la conciencia se pierda alguna vez en el mundo o que se lleve a sí misma a un estado de quietud" (Luhmann 1997: 35). Esta posibilidad universal de oscilación es lo que Kant identificó como autonomía de la voluntad, de lo cual derivó un reino de los fines como máxima moral (2002b). La oscilación entre auto y heterorreferencia, sin embargo, no actúa solo para la voluntad, sino también para otros rendimientos de la conciencia, como la percepción, el sentimiento, el pensamiento (es decir, habría autonomía de todas ellas). Pero a la vez, esa misma oscilación no asegura cuál es la distinción que el observador aplicará, ni cómo articulará las paradojas derivadas de cada rendimiento; por ejemplo: se puede querer que sí, percibir que no, sentir la necesidad y pensar en posponer todo. La bifurcación de auto y heterorreferencia genera esta oscilación (autonomía, libertad), pero su manejo se construye en la observación.

En el plano de la observación, emergen las construcciones de la conciencia. Cualesquiera sean las cogitaciones (percepción, pensamiento, sentimiento, voluntad) ellas tienen como base la referencia a sí mismas mediante un nuevo evento (autorreferencia) o la referencia al mundo (comunicación) (Luhmann 2005d, 2005e). El ultramundo permanece inaccesible en el sentido kantiano como Ding an Sich, pero presupuesto en la continuidad de la operación reconocible en la observación (por ello se puede saber que no hay que saltar del balcón para bajar más rápido, o que el Río Amarillo puede crecer a pesar de la fuerza mental de un constructivismo ingenuo).

Revista Mad. N²3, Septiembre de 2010. Departamento de Antropología. Universidad de Chile http://www.revistamad.uchile.cl/23/mascareno_02.pdf 
Auto y heterorreferencia accionan la irritabilidad (observación) de la conciencia sobre las propias cogitaciones o sobre las cogitaciones (propias) acerca del mundo. En el primer caso se forman recursivamente identidades acerca del sí mismo, o ultimate concerns en el sentido de Archer (2003); en el segundo, ellas son operativamente monitoreadas (reflexivamente, si se quiere) en su ajuste a las condiciones de la comunicación. Esta es la potencialidad de la diferencia entre el ' $\mathrm{I}$ ' y el 'Me' de Mead, figura que M. Archer (2003) pone en términos de diversos momentos de reflexividad del self por medio de la operación de un turn-taking, en el que el self aparece como sujeto (autorreferencia) y como objeto (heterorreferencia). Mediante esa operación (sea se le llame autorreferencia/heterorreferencia, intencionalidad de la conciencia, reflexividad) de registro de identidades y de desviaciones surgidas en la observación heterorreferente del mundo, emergen temporalmente condensaciones que estructuran la operación de la conciencia en términos de expectativas y que funcionan como diferencias directrices de nuevas observaciones (Luhmann 2005c). Ellas pueden ser cognitivas (del tipo 'si, entonces' -heterorreferencia) o normativas (del tipo 'es un deber' -autorreferencia). El segundo caso mueve a la continuidad en la expectativa (mantención de ultimate concerns, refuerzo de la identidad); el primero la cognitiviza, es decir, le abre la alternativa de reorganización ante las posibilidades del mundo que se ha construido en la observación. En ambos casos se trata de mecanismos de autorregulación de la conducta y de lo que se experimenta en ella. Y, como siempre, se puede oscilar entre auto y heterorreferencia, es decir: ni el mundo (el problema de la facticidad) ni la norma (el problema de la validez) son o deben ser siempre iguales. Aunque sin duda, la observación normativa se resiste más al cambio, especialmente cuando, por un lado, se vivencia que las condicionalidades del mundo cambian día a día, sistema a sistema, sujeto a sujeto, y, por otro, se actúa en consecuencia (o no), o se vivencia cómo otros lo hacen (o no). Con ello ya se entra en el terreno del acoplamiento entre la observación psíquica y la social. Primero, sin embargo, es preciso revisar operación social.

\section{Construct this!: la operación social}

El caso especial de la operación social tiene como último elemento la comunicación (no las cogitaciones de la conciencia). Aun cuando no se trata simplemente de un acto, este es su unity act. Es sabido que Luhmann (1984) distingue para la operación de la comunicación entre tres selecciones: información, conducta de notificación y comprensión. Sin embargo, quiero proponer aquí que en un nivel fundamental (la ultrarealidad de la operación social), se trata solo de la iteración de una distinción: la distinción entre información y notificación. La información constituye la heterorreferencia de la operación (aquello que el sistema busca expresar) y la notificación su autorreferencia (el modo en que el sistema lo expresa). Sin aún introducir las figuras de alter y ego, la estructura iterativa tiene su origen en la oscilación entre auto y heterorreferencia de la manera siguiente:

a. La heterorreferencia basal de la operación genera una indicación acerca de un estado del sistema (un fenómeno, información); la autorreferencia permite condensar esta información en una notificación que (re)itera la información en un formato observable (acción, habla, movimiento).

b. La heterorreferencia permite al sistema la observación de la notificación y su contenido informativo; la autorreferencia permite al sistema distinguir entre notificación e información (comprensión).

c. La heterorreferencia selecciona una información; la autorreferencia hace posible condensar esa información en una notificación de aceptación o rechazo de la 
información previa en un formato observable (acción, habla, movimiento) que (re)itera la información de aceptación o rechazo.

Tanto como en el caso de la conciencia, la oscilación operativa de auto y heterorreferencia genera apertura en la estructura selectiva, y se puede agregar en este caso: genera iteración en cada oscilación, es decir, la información se altera en su reiteración en la forma de la notificación; o dicho de otro modo: itera re-iteradamente. Esto es ultrarealidad de la operación social; no es construida por el observador, es su punto ciego, condición de posibilidad de observar. En este sentido vale aquí también el mandato imposible: construct this! Lo que el observador construye es la coordinación selectiva de la comunicación como acuerdo o desacuerdo, como consenso o disenso, como unidad o diferencia; construye, por tanto, una ficción de no iteración, de no oscilación que debe entenderse como real para no caer en la recursión ad infinitum de cada evento y no tener que constatar continuamente si la notificación es la reconstitución de la información por otros medios o no. Si la comunicación no suspendiera esta problematización en sus distinciones (o si no las dejara, al menos, al análisis sistémico), habría permanecido para siempre en la primera oscilación entre auto y heterorreferencia, en la primera iteración entre información y notificación. Es decir, habría tratado de encontrar el Ding an Sich de la operación y habría implosionado en ella; o se habría conducido a sí misma a su propio estado de quietud en la paradoja de una re-entry idéntica a sí misma.

Para direccionar esta iterabilidad de la comunicación, y como puntos de anclaje discretos de la continuidad de la operación, se requieren las figuras de alter y ego. En la fórmula canónica se indica: alter selecciona una información y la notifica; ego observa y distingue la notificación de su contenido informativo, acepta o rechaza, y con ello enlaza un evento comunicativo a otro (Luhmann 1984). De este modo, la oscilación auto/heterorreferencia puede posicionalizarse y temporalizarse en el propio proceso de comunicación. Alter y ego son las posiciones, y la temporalidad difiere si se observa el proceso analíticamente o como unidad. Analíticamente, es decir, como secuencia de selecciones, el tiempo corre de alter a ego: alter es antes y ego es después - primero información, luego notificación, luego comprensión. En la unidad del proceso el tiempo emerge en sentido inverso, pues si la comprensión de ego cierra la selectividad de la comunicación (la constituye como tal y la reabre a la vez a otro ciclo), entonces la comunicación no emerge sino hasta ese evento, no antes ni después: 'antes' es aún potencialidad de sentido, 'después' ya es otra comunicación; y solo cuando la comunicación emerge se puede mirar atrás en su interior y distinguir cómo alter distingue entre información y notificación. Por ello, en la comunicación vista como unidad, ego es antes y alter después, o si se quiere decir de otro modo: primero se comprende y después se selecciona y se notifica.

La introducción de las figuras de alter y ego también permite direccionar la contingencia (oscilación) de la comunicación por medio de la doble contingencia. Doble contingencia significa apertura de la estructura selectiva a dos instancias de selección: alter y ego. Luhmann (1998: 17) habla en este caso de una duplicación de la estructura de selección: "La duplicación comprende toda la estructura: el potencial generalizado para concebir hechos como selecciones que implican negaciones, para negar estas negaciones y para reconstruir otras posibilidades [...] El potencial es universal para cada sujeto y es un aspecto de las constituciones significativas de su entorno, de manera que ego tiene que identificar a alter como otro sujeto en su mundo, y viceversa. La doble contingencia descansa en el hecho de que la contingencia es subjetiva y universal a la vez". La selección supone distinguir y distinguir es indicar. La indicación deja un lado no indicado, no actualizado, latente, 
negado, que puede ser negado como negación 'después', es decir, actualizado para construir nuevas distinciones que indican otras actualizaciones que suponen otras negaciones que pueden ser negadas; y así sucesivamente. Este es el potencial disponible para alter y para ego, y si ello es así, la doble contingencia hace la selectividad de alter selectivamente disponible para ego.

Como en el caso de la conciencia, la comunicación también crea condensaciones en torno a la recursión de selecciones de alter y ego. Se trata de las expectativas cognitivas y normativas (Luhmann 2005f). Ellas controlan a nivel social la oscilación operativa de auto y heterorreferencia y regulan las paradojas que surgen del hecho que la selectividad de alter sea selectivamente disponible para ego (por ejemplo, que una posición de observación sea ocupada por valores distintos, o que valores distintos indiquen la misma posición -Luhmann 1993). Las expectativas permiten el cambio de direccionalidad de la comunicación (morfogénesis) por medio del aprendizaje del mundo (expectativas cognitivas), y permiten la mantención de direccionalidad de la comunicación (morfoestásis) por medio de la resistencia al aprendizaje del mundo (expectativas normativas). Es decir, las expectativas habilitan y constriñen las posibilidades de comunicación, la abren a la novedad y limitan a sobreabundancia de novedad que surge día a día, sistema a sistema, sujeto a sujeto. Los medios simbólicos y sistemas son el nivel de construcción mayor de la comunicación; son un entrelazamiento de expectativas cognitivas y normativas de distinto tipo, con asiento en la ultrarealidad de la operación social y con una realidad significativa y estructural que permite sostener el sentido de mundo como un horizonte de potencialidades y actualizaciones oscilante y contingente, subjetivo y universal a la vez.

Para ello, la ultrarealidad de la operación psíquica y social permanecen separadas como entorno una de otra, pero sus observaciones se acoplan de algún modo, se coconstruyen en base sus respectivas operaciones. En este plano, el constructivismo sistémico alcanza su mayor despliegue como construcción de realidad social.

\section{El acoplamiento de construcción psíquica y social}

La autonomía de sistemas psíquicos y sociales, como se ha visto hasta aquí, no es un presupuesto o una construcción del observador. Ella descansa en modos de operación diferenciados que por su inaccesibilidad para la observación psíquica y social se constituyen en garantía de realidad de la observación. En ambos casos la operación se constituye por una oscilación entre autorreferencia y heterorreferencia. Con ello, tanto sistemas psíquicos y sociales pueden referirse a sí mismos o a su entorno. La heterorreferencia de los sistemas psíquicos es la comunicación; la de la comunicación es el sistema psíquico, o la conciencia en términos tradicionales. Puesto que se trata en ambos casos de heterorreferencia, el sistema psíquico descrito por la comunicación no es el sistema psíquico tal y como su propia autorreferencia lo indica, y viceversa. Ambos aparecen intercambiadamente como eventos en la oscilación del otro, eventos que pueden coincidir temporalmente sobre una base de aleatoriedad. Este carácter de aleatorio de la coincidencia en la referencia mutua de sistemas psíquicos y sociales es, sin embargo, muy débil para estructurar una interrelación. Para ello hay que probabilizarla por medio de un acoplamiento. El acoplamiento requiere que los sistemas pongan a disposición elementos de su complejidad para una interrelación duradera (Luhmann 2007). Quiero proponer aquí que la comunicación pone a disposición de los sistemas psíquicos información que estos reconstruyen en la forma de vivencias, y que el sistema psíquico pone a disposición de los sistemas sociales intencionalidades que estos reconstruyen como conductas de notificación o acciones. 
En este sentido puede hablarse aquí de un acoplamiento como inter-irritación de sistemas: la información irrita la conciencia como vivencia, esto es, como heterorreferencia tanto de la operación psíquica como de la social; y la intencionalidad irrita la operación social en modalidad de acción, esto es como autorreferencia de ambas operaciones. Con ello el acoplamiento se sostiene en su propia heterorreferencialidad (información-vivencia) y en su propia autorreferencialidad (intencionalidad-acción). Por esta razón la vivencia es atribución al entorno y la acción al sistema (Luhmann 2005g). Vivencia es una atribución al entorno porque el estado interno que ella provoca en alter o ego no puede conseguirse por introspección, es motivado heterorreferencialmente por una información en la comunicación, es decir, en el entorno del sistema psíquico. Acción es una atribución al sistema, porque la información en cuanto tal, una vez vivenciada, puede permanecer como vivencia interna de alter o ego sin que sea de algún modo actualizada; es decir, puede mantenerse como latencia disponible al futuro eternamente. Hay que actuarla enactuarla dice Varela (1990) - para que tenga valor de conectividad comunicativa y motive a la comprensión en términos de aceptación o rechazo. En tal sentido, alter puede vivenciar y actuar, y ego puede hacer lo mismo.

Como se aprecia, esta es una versión de mayor alcance empírico de la duplicación de la estructura de selectividad de la doble contingencia. Con ella, alter y ego pueden encontrarse en ciertos escenarios sociales problemáticos dignos de atención y probar si con determinado acoplamiento de vivencias y acciones de uno y otro, es posible desparadojizar la situación y estabilizar una vía para direccionarla en el futuro, cuando aparezca nuevamente. Vista desde el self (alter o ego), esta situación se articula del modo siguiente: si puedo aceptar, de un momento a otro (antes/después), una información como vivencia o si requiero observar una acción para incorporarla de modo significativo como evento vivenciado. Algunas de estas situaciones sociales improbables son las que han dado origen a los medios de comunicación simbólicamente generalizados (ver Luhmann 2007: 258ss); estas son:

- Implantar un saber nuevo, inaudito; apartarse de un conocimiento existente o criticarlo - verdad

- Continuidad de contacto entre sistemas psíquicos ante doble contingencia y clausura operativa - valores

- Apoyo a visión propia del mundo a través de otro -amor

- Especificación de interés por medio de acción sobre elementos en los cuales el observador pueda tener interés - propiedad/dinero

- Reactivación de posibilidades descartadas-arte

- Actuar de alter consiste en decisión sobre actuar de ego - poder/derecho

Estas situaciones no son las únicas posibles; son las que la propia selectividad ha construido para resolver problemas que ella misma se crea; problemas que ella además considera dignos de resolver, pues también se puede vivenciar la acción de alguien que riega las plantas, pero la selectividad no ha entendido que ello sea digno de la formación de una constelación simbólica o de un sistema social. Mediante el acoplamiento de vivencias y acciones, la selectividad de la conciencia y de la comunicación pueden irritarse mutuamente y seleccionar en la comunicación opciones para las cuales alter y ego puedan encontrar motivación sin tener que negociar o acordar siempre desde cero la legitimación de cualquier situación social específica. Ello permite concentrarse en problemas donde la legitimidad se pone en duda porque no se ajusta a la expectativa normativa de alter o ego, por ejemplo en el plagio en la ciencia o en los riesgos de las innovaciones técnicas, en la vulneración de derechos fundamentales o en situaciones de criminalidad, en casos de explotación en el trabajo 
o de incompetencia, en la hipertextualidad del arte o en la invasión de esferas privadas, en la infidelidad en el amor o en la expansión de prácticas sexuales, en la imposición de doctrinas o en las controversias morales. Esto hace posible también que emerjan situaciones nuevas para sustentar expectativas normativas constantemente defraudadas; por ejemplo: un medio simbólico de la cooperación como transmisión de libertad de inclusión desde la acción de alter a la vivencia de ego para regular los efectos de la exclusión social del sistema económico, jurídico y político (Mascareño 2007), o la diferenciación del medio amor en pasión y placer como satisfacción de la vivencia individual sin búsqueda de apoyo a la propia visión de mundo (Luhmann 2008), o la sistematización de los valores desde la semántica de derechos humanos a la operatividad de derechos fundamentales de nivel constitucional para una ciudadanía cosmopolitita (Mascareño/Chernilo 2009; Chernilo 2009).

Para todos estos casos siempre hay dos posibilidades: o la norma se puede cognitivizar y se ajusta al mundo, o se mantiene contrafácticamente. Por ello ni los medios simbólicos ni la estructuras sistémicas que se despliegan en paralelo son formas de presión hidráulica sobre las conciencias: primero, por la propia autonomía operativa de cada nivel que fija un límite a esto; segundo, por que la acción y la vivencia siempre pueden moverse más allá de las estabilizaciones estructurales o simbólicas; y tercero, porque para mucho de lo socialmente estabilizado, la selectividad comunicativa cuenta con la motivación individual.

No hay, entonces, una oposición entre sistema social y subjetividad, sino un acoplamiento por la vía de vivencias y acciones que permite unidad y diferencia, continuidad y cambio, morfogénesis y morfoestásis entre conciencia y comunicación; que permite, en suma, distinguir entre la contribución de los sistemas psíquicos a la emergencia de lo social y la contribución de los sistemas sociales a la emergencia de los sistemas psíquicos. Esta realidad por cierto que es construida por la recurrencia de esquemas de distinción y sus múltiples variaciones, pero carece de sentido considerarla un simulacro de algo que guardaría la verdad de las relaciones sociales como última ratio y frente lo cual solo cabe medir la distancia que nos separa para emprender la tarea heroica de mover la realidad psíquica y social hacia allá, sea que esta verdad se esconda en el plano de la objetividad absoluta del ultramundo, o en un futuro de utopías de realización trascendental o histórica. La continuidad del ultramundo se advierte en su autonegación a la observación, negación que es a la vez garantía para la observación de que ella no flota perdida y a la deriva en la nada ni que tampoco es la eterna reiteración de sí misma. Y la apertura del futuro se asienta en la oscilación de auto y heterorreferencia como contingencia de cada selección - 0 , por último, en el hecho que incluso hoy existen distintos proyectos trascendentales o históricos de realización de fines: mientras ego y alter puedan seguir optando por uno u otro, las opciones siguen abiertas.

\section{Constructivismo realista}

Construct this!: la operación sistémica es real (ultrareal) y es el punto ciego del observador. El observador construye un mundo significativo también real gracias a esta ceguera de su operación, de otro modo se perdería en ella, en el caos de sensaciones del noumeno kantiano: "resulta que el uso de las categorías no puede en modo alguno rebasar los límites de los objetos de la experiencia; a los entes sensibles corresponden ciertamente entes inteligibles, y aun puede haber entes inteligibles con los cuales nuestra facultad sensible de intuir no tenga ninguna relación; pero nuestros conceptos del entendimiento, como meras formas del pensamiento, para nuestra intuición sensible, no alcanzan a esos entes; lo que llamamos noumeno debe, pues, 
como tal, ser entendido sólo en sentido negativo" (Kant 1992[1781]: 423). La inaccesibilidad de la cosa en sí es en Kant el punto de partida para encontrar en el entendimiento lo que puede ser conocido en forma de fenómeno. La consecuencia es el sujeto trascendental racional en sentido cognitivo y normativo.

En la teorías realistas de la actualidad, la operación teórica no es muy distinta: "No hay acceso directo a los 'hechos duros' de la vida social, al menos para la gran mayoría de nosotros que no podemos aceptar la doctrina, ya desacreditada, de una percepción inmaculada. Al describirla en términos particulares, estamos de hecho definiendo aquello que debe ser explicado" (Archer 2009: 50). El realismo de M. Archer tampoco puede entenderse como un redescubrimiento de la cosa en sí, como una especie de substancialismo sociológico del siglo XXI. Realismo es encontrar la más fina adecuación entre los conceptos descriptivos de la realidad social y los conceptos explicativos, esto es, entre explanandum y explanans, evitando con ello que lo implicado en el explanandum rebase los límites de lo contenido en el explanans. Si el explanandum describe la sociedad como compuesta de individuos, en el explanans no pueden introducirse criterios por la puerta trasera que presupongan la autonomía de estructuras sociales y su influencia sobre los individuos. Para ello habría que modificar el explanandum. Del mismo modo, si se ha supuesto un explanandum de lo social como producto de una estructura de alcance universal, el explanans no puede ser inconsistente con esta visión e incorporar procesamientos autónomos individuales. Si el explanandum, en cambio, describe el fenómeno a explicar como autonomía de niveles, el explanans debe considerar en su explicación la interrelación de ellos. Esto es realismo. Puesto que no hay acceso directo a los 'hechos duros' de la vida social, 'realismo' es consecuencia explicativa con la forma en que el mundo es descrito; consecuencia entre fenómeno y juicio se diría kantianamente, entre explanandum y explanans en los términos de Archer.

Al explanandum es a lo que Archer llama ontología social: la descripción del mundo. Si en las posiciones tradicionales del individualismo y el estructuralismo (colectivismo) aparecía serendipia, entonces la opción no podía ser solo mejorar el método explicativo sin cambiar la descripción. Ella tendría que indicar que algo no funcionaba en la opción ontológica asumida, es decir, debía indicar que la realidad era distinta, pues el dato anómalo apunta a propiedades autónomas no reductibles a los principios explicativos de los que se dispone. Un ejemplo de esto es el 'verdadero individualismo' de Hayek, para quien el orden espontáneo emergente es un "resultado imprevisto de las acciones individuales" (1986: 8). El explanans individualista (la sociedad la hacen la interacción de individuos) es estirado al máximo para ajustarlo al dato anómalo (el orden espontáneo). Con el dato anómalo, sin embargo, se dice que el método encontró 'algo' que no era producto directo de la interacción individual (que contradecía al explanans), pero no se juzga suficiente razón para cambiar el explanandum, esto es, la concepción ontológica (por ejemplo a una fórmula que bien pudo ser: hay propiedades irreductibles de lo individual y también de lo social), sino que se paradojiza el explanans de la creación individual interactiva de sociedad con la fórmula del 'resultado imprevisto' de ella. Así, el explanans afirma y niega a la vez lo que sostiene: $(+)$ la sociedad es la creación interactiva de individuos; (-) la sociedad es más que la creación interactiva de individuos. El compromiso ontológico pudo más que la constatación de su insuficiencia; al operar de este modo no se cumple finalmente con el dictum realista: "Lo que pensamos acerca de la realidad social no puede ser una cosa distinta de aquello que encontramos en ella" (Archer 2009: 57). O dicho de otro modo menos provocativo: si el explanans indica algo no contenido en el explanandum, entonces se debe cambiar el explanandum. Realismo, entonces, no es objetivismo. Es 
desontologización de los 'hechos duros' (noumena), y ontología como mecanismo regulativo de descripción y explicación de lo social.

El constructivismo sistémico no está lejos de esto: "El efecto de esta intervención de la teoría de sistemas puede ser descrito como desontologización de la realidad. Esto no significa que la realidad sea negada, pues entonces no habría nada que pudiera operar, nada que pudiera observar y nada que pudiera ser aprehendido con distinciones [...] Los sistemas cognoscentes son sistemas reales (empíricos, esto es observables) en un mundo real. No podrían, bajo ningún punto de vista, existir sin mundo, y tampoco conocer. El mundo es para ellos, sin embargo, cognitivamente inaccesible" (Luhmann 2005a: 35-36, 39). Cognitivamente inaccesible es también el noumeno y los 'hechos duros', pero ambos son igualmente garantía para el fenómeno de la razón trascendental, para la regulación de la relación explanans-explanandum del realismo, y para la unidad de la diferencia entre operación y observación que está en la base del constructivismo sistémico. Los programas teóricos que surgen en estos casos son, por cierto, distintos: un proyecto racionalista de teoría social con sustanciales consecuencias normativas, en el primer caso; un proyecto realista de teoría social como interrelación entre las propiedades irreductiblemente autónomas de la estructura y de la agencia; y un proyecto constructivista de teoría social que afirma la operación autónoma y acoplada de lo social y lo psíquico. Sin embargo, lo que comparten es la distinción entre una ultrarealidad que es inaccesible a la observación (razón, explicación) y una realidad significativa y social que no tiene el estatuto de realidad de la ultrarealidad, pero que es la única de la que los observadores (sujetos, agentes) disponen. Es, por tanto, su realidad.

La ontología social del constructivismo sistémico es un mundo significativo como horizonte de posibilidades de selección. Pero la selección presupone complejidad y la complejidad, contingencia. La ontología social del constructivismo sistémico (su explanandum) es, así, la de un mundo contingente que oscila en distinciones para el manejo de esa contingencia. Su preocupación (explanans) es, por tanto, cómo las distinciones oscilan para mantener la contingencia (morfogénesis) y cómo se estabilizan para manejarla (morfoestásis). El resultado de ello es una ficción de realidad que no puede operar como ficción porque la operación es inaccesible para el observador. El observador puede distinguir, acoplar distinciones con otros observadores y disentir mediante nuevas distinciones para crear el mundo significativo. Todo, en realidad, depende de ese mundo. RM

\section{Referencias}

Archer, M. (2003): Structure, Agency and the Internal Conversation. Cambridge: Cambridge University Press.

Archer, M. (2009): Teoría social realista. El enfoque morfogenético. Santiago: Ediciones Universidad Alberto Hurtado.

Berkeley, G. (2008): Philosophical Writings (ed. D. Clarke). Cambridge: Cambridge University Press.

Beyme, K.v (1994): Teoría política del siglo XX. Madrid: Alianza.

Chernilo, D. (2009): Nacionalismo y cosmopolitismo. Santiago: Ediciones Universidad Diego Portales. 
Durkheim, E. (1985): La división del trabajo social. Barcelona: Planeta-Agostini. Hayek, F. v. (1986): Individualismo: el verdadero y el falso (1-28). Estudios Públicos 22.

Husserl, E. (2005). Meditaciones cartesianas. México D.F.: Fondo de Cultura Económica.

Kant, I. (1992): Crítica de la razón pura. Buenos Aires: Losada.

Kant, I. (2002a): Filosofía de la historia. México D.F.: Fondo de Cultura Económica.

Kant, I. (2002b): Fundamentación para una metafísica de las costumbres. México D.F.: Fondo de Cultura Económica.

Luhmann, N. (1984): Soziale Systeme. Frankfurt: Suhrkamp.

Luhmann, N. (1993): Die Paradoxie der Form (197-212). En D. Baecker (ed.): Kalkül der Form. Frankfurt: Suhrkamp.

Luhmann, N. (1997): Die neuzeitliche Wissenschaften und die Phänomenologie. Wien: Picus Verlag.

Luhmann, N. (1998): Los medios generalizados y el problema de la doble contingencia (9-73). En Teoría de los sistemas sociales (artículos). México D.F.: Universidad Iberoamericana.

Luhmann, N. (2005a): Das Erkenntnisprogramm des Konstruktivismus und die unbekannt bleibende Realität (31-57). En Soziologie Aufklärung 5. Wiesbaden: VS Verlag.

Luhmann, N. (2005b): Die operative Geschlossenheit psychischer und sozialer Systeme (26-37). En Soziologie Aufklärung 6. Wiesbaden: VS Verlag.

Luhmann, N. (2005c): Die Autopoiesis des Bewußtseins (55-108). En Soziologie Aufklärung 6. Wiesbaden: VS Verlag.

Luhmann, N. (2005d): Wie ist Bewußtsein an Kommunikation beteiligt (38-54). En Soziologie Aufklärung 6. Wiesbaden: VS Verlag.

Luhmann, N. (2005e): Die Form ,Person' (137-148). En Soziologie Aufklärung 6. Wiesbaden: VS Verlag.

Luhmann, Niklas (2005f): Die Weltgesellschaft (63-88). En Soziologische Aufklärung 2. Wiesbaden: VS Verlag.

Luhmann, N. (2005g): Erleben und Handeln (77-92). En Soziologie Aufklärung 3. Wiesbaden: VS Verlag. 
Luhmann, N. (2007): La sociedad de la sociedad. México D.F.: Herder, Universidad Iberoamericana .

Luhmann, N. (2008): Liebe. Eine Übung. Frankfurt: Suhrkamp.

Maturana, H. (1982): Erkennen: Die Organisation und Verkörperung von Wirklichkeit. Ausgewäh/te Arbeiten zur biologischen Epistemologie. Braunschweig/Wiesbaden: Friedr. Vieweg \& Sohn.

Mascareño, A. (2006): Ethic of contingency beyond the praxis of reflexive law. Soziale Systeme 12(2): 274-293.

Mascareño, A. (2007): Sociología de la solidaridad. La diferenciación de un sistema global de cooperación (35-68). En M. Arnold y D. Thumala (eds.): Colaboración, cultura y desarrollo. Revista MAD, Edición Especial 2.

Mascareño, A. y Chernilo, D. (2009): Obstacles and Perspectives of Latin American Sociology: Normative Universalism and Functional Differentiation. Soziale Systeme 15(1): 72-96.

Nassehi, A. (2006): Der soziologische Diskurs der Moderne. Frankfurt: Suhrkamp.

Parsons, T. (1970): The social system. London: Routledge \& Kegan Paul.

Parsons, T. (1978): Action Theory and the Human Condition. New York: The Free Press.

Parsons, T. (2007): American Society. A Theory of the Societal Community. Boulder: Paradigm Publishers.

Simmel, G. (1992): Soziologie. Frankfurt: Suhrkamp.

Stichweh, R. (2003): Raum und moderne Gesellschaft. Aspekte der sozialen Kontrolle des Raums (93-102). En T. Krämer-Badoni y K. Kuhm (eds.), Die Gesellschaft und ihr Raum. Raum als Gegenstand der Soziologie. Opladen: Leske + Budrich.

Varela, F. (1990): Conocer. Barcelona: Gedisa.

Weber, M. (1992): Economía y sociedad. México D.F.: Fondo de Cultura Económica. 